\title{
In Vivo Comparison of Three Human Acellular Dermal Matrices for Breast Reconstruction
}

\author{
PHAM NGOC CHIEN ${ }^{1}$, XIN RUI ZHANG ${ }^{1,2}$, DONMEZ NILSU ${ }^{1}$, OMAR FARUQ ${ }^{1}$, \\ LE THI VAN ANH ${ }^{1}$, SUN-YOUNG NAM ${ }^{1}$ and CHAN YEONG HEO ${ }^{1,2}$ \\ ${ }^{1}$ Department of Plastic and Reconstructive Surgery, \\ Seoul National University Bundang Hospital, Seongnam, Republic of Korea; \\ ${ }^{2}$ Department of Plastic and Reconstructive Surgery, College of Medicine, \\ Seoul National University, Seoul, Republic of Korea
}

\begin{abstract}
Background/Aim: Acellular dermal matrices $(A D M s)$ have become popular in implant-based breast reconstruction. The aim of this study was to compare three commonly used ADM products in vivo in an animal model. Materials and Methods: The nucleic acid content (residual double-stranded DNA) and the levels of the remaining growth factors after decellularization were measured for each ADM. Cytocompatibility with ADMs was documented using NIH 3 T3 mouse fibroblast cells. In vivo, the implanted ADMs were histologically evaluated at 1, 2, 3, and 6 months $(n=5)$ using male 8-week-old Sprague-Dawley rats. Results: Fibroblasts grew in the SureDerm HD and DermACELL with no cytotoxicity. In a rat model, SureDerm $H D$ and DermACELL incorporated more readily into the surrounding host tissue, as measured by rapid cell influx and collagen deposition, and showed more delayed tissue remodeling with decreased matrix metalloproteinases levels compared to AlloDerm. Conclusion: SureDerm HD and DermACELL can be used as biological materials for breast reconstruction.
\end{abstract}

Acellular dermal matrix (ADM) is a tissue graft obtained from cadaveric skin, which is widely used in tissue reconstruction, particularly burn injuries, abdominal wall repair, dural repair,

This article is freely accessible online.

Correspondence to: Sun-Young Nam, Department of Plastic and Reconstructive Surgery, Seoul National University Bundang Hospital, Seongnam 13620, Republic of Korea. Tel: +82 317877222, e-mail: $99261 @$ @nubh.org (S.Y.N); Chan Yeong Heo, Department of Plastic and Reconstructive Surgery, College of Medicine, Seoul National University, Seoul 03080, Republic of Korea. Tel: +82 317877222, e-mail: lionheo@snu.ac.kr

Key Words: Acellular dermal matrix, decellularization, host response, extracellular matrix, collagen. tympanic membrane replacement and breast reconstruction (18). Decellularization technique has been employed for the preparation of ADM, followed by terminal sterilization to preserve the biochemical and structural components of the extracellular skin matrix. ADMs are increasingly used for skin graft in tissue engineering since they contain complex proteins, signaling cascades, and biomolecules for restoration of damaged tissue. The presence of collagen and elastin maintain favorable tensile strength and elasticity, while proteoglycans and laminin induce angiogenesis and connective tissue binding, respectively. Besides, they provide a biocompatible environment and promote reepithelization, neovascularization, and fibroblast infiltration without inducing immune response at implant site (1, 9-11). Furthermore, ADM degradation results in the release of growth factors and biochemical signals similar to the native tissue, maintaining cellular behavior in terms of attachment as well as growth. Therefore, ADM implantation into the human body influences host remodeling responses such as rapid ingrowth of blood vessels, cell migration, proliferation, differentiation and support for regenerative healing and prevent scar tissue formation after implantation (12-15).

The application of ADMs as breast implants was reported in 2005 by Breuing and Warren using AlloDerm sling (LifeCell Corporation, Branhchburg, NJ, USA) to directly implant 10 patients undergoing bilateral mastectomy (16). By single stage alloplastic reconstruction, they showed that their technique "avoids or shortens the tissue expansion/implant reconstructive process, avoids mastectomy flap contraction during the latency period of expansion, and provides an additional option for immediate, single stage breast implant reconstruction". Furthermore, this approach has significantly influenced surgery and become standard of care in breast reconstruction after mastectomy. The benefits of using AMD as a sling are useful for immediate prosthetic breast reconstruction and can provide a tissue expander or a larger implant pocket with extremely low 
complication rate and long-term safety, while being time- and cost-effective. In addition, the use of ADMs not only helps to reconstruct pleasing breasts with natural soft tissue appearance but also facilitate speedy completion of the reconstruction (1619). Maintaining three-dimensional ultra-structure and conserving the complex composition of ADMs during decellularization is challenging at current time. It is well recognized that all decellularization techniques disrupt the architecture of tissue to some degree and affect surface structure and composition (20). In addition, insufficient removal of cellular and nuclear components is frequently observed during decellularization. As a result, several types of major complications have occurred including immunogenic reaction, fibrotic capsular formation, inflammation, and scar tissue formation (21-23). Therefore, it is crucial to choose the most applicable implants among the available biological scaffolds and consider the factors influencing host response during the clinical application.

The current work was focused on comparing the performance of three commercial human ADMs, namely AlloDerm, SureDerm HD and DermACELL. Their biochemical composition as well as their effect on in vitro cell viability were evaluated. Moreover, these commercial ADMs were implanted in vivo in rat models of breast reconstruction to determine host tissue response. Furthermore, quantitative histomorphologic metrics were established to recognize the inflammatory and tissue remodeling responses in vivo.

\section{Materials and Methods}

Materials. Three samples of each of the following ADMs were used in each experimental test; 1) AlloDerm from LifeCell Corp (Branchburd, NJ, USA); 2) SureDerm HD from Hans Biomed Corporation (Seoul, Republic of Korea); 3) DermACELL from LifeNet Health (Virginia Beach, VA, USA). Paraformaldehyde (4\%) was obtained from KCFC (Ansan, Republic of Korea). Hematoxylin and eosin (H\&E), xylene, dehydration agent (ethanol), and $\mathrm{HCl}$ acid were supplied by Duksan Pure Chemicals (Ansan) and paraffin was from Merck (Branhchburg, NJ, USA). Modified Mayer's H\&E Y solution was from Richard-Allan Scientific (Kalamazoo, MI, USA) and ammonia solution was from JunsieChemical (Tokyo, Japan). In order to determine collagen synthesis after ADM implantation by Masson's trichrome (MT) staining, aniline blue solutions, Biebrich scarlet-acid fuchsin, phosphotungstic acid and phosphomolybdic acid were obtained from Sigma-Aldrich (St. Louis, MO, USA) and acetic acid (1\%) was supplied by Duksan Pure Chemicals (Ansan). For Immunofluorescent Staining, antigen-retrieval solution was acquired from Dako (Glostrup, Denmark). The primary antibodies anti-hTGF- $\beta 1$ (ab92486, Abcam, Cambridge, MA, USA), antiVEGF (ab46154, Abcam) and anti-bFGF- $\beta 1$ (MAB233, R\&D systems, Minneapolis, MN, USA) were used in this study.

Decellularization assessment. The level of decellularization was determined for AlloDerm, SureDerm HD, and DermACELL based on the established criteria such as 4',6 diamidino-2-phenylindole (DAPI) stain and H\&E staining, to determine the presence of DNA and intact cells and nuclei, respectively. Further, the size of existing DNA fragments was determined by gel electrophoresis methods as well as by quantifying double stranded DNA (dsDNA) by the Quant-IT PicoGreen assay (Invitrogen, Carlsbad, CA, USA).

Biochemical analysis. Concentrations of vascular endothelial growth factor (hVEGF), transforming growth factor- $\beta 1$ (hTGF- $\beta 1$ ), and basic fibroblast growth factor (bFGF) were determined using human VEGF immunoassay, human TGF- $\beta 1$ immunoassay and human FGF basic Immunoassay (all R\&D Systems) as described at the manufacturer's protocol. All examinations were performed in duplicate.

In vitro cytocompatibility analysis. The mouse fibroblast cell line NIH 3 T3 was cultured in Dulbecco's modified Eagle's medium containing $10 \%$ fetal bovine serum, $1 \%$ of penicillin-streptomycin and maintained in an incubator at $37^{\circ} \mathrm{C}$ in $5 \% \mathrm{CO}_{2}$ until $80 \%-90 \%$ confluence. All ADMs used for cell seeding were cut uniformly 2.5 $\mathrm{cm}^{2}$ and subsequently placed in 6 well plates, hydrated according to description on the manufacturer's protocol and incubated in the media at $37^{\circ} \mathrm{C}, 5 \% \mathrm{CO}_{2}$ for $15 \mathrm{~min}$. Then, the media were discarded and the cells were seeded at a density of $10^{6}$ cells per $1.5 \mathrm{~cm}^{2}$. After 7 days of culture, the cells attached to the grafts were fixed with $10 \%$ formalin for $24 \mathrm{~h}$ and embedded in paraffin blocks to prepare slices for H\&E staining. The cell phenotype and cell infiltration into the ADM substrate were analyzed.

In vivo experiment. Eight-week-old Sprague Dawley rats were used for the in vivo experiment. The animals weighing 250-300 g were kept to $12 / 12 \mathrm{~h}$ light/dark cycle and maintained in pathogen-free conditions with free access to food and drinking water. The animal experiments were approved by the Institutional Animal Care and Use Committee of Bundang Seoul National University Hospital (approval number: BA-1802-241-014-08) and all procedures were conducted based on NIH guidelines for the Care and Use of Laboratory Animals. Twenty animals for each group were implanted with the AlloDerm, SureDerm HD, or DermACELL separately. In order to implant ADM, the rats were anesthetized using inhalation of isoflurane (Hana Pharm, Seoul, Republic of Korea). After shaving the dorsal region of the three groups of rats, $70 \%$ alcohol and betadine were applied to the surgical site. \#15 scalpel blades were used for implantation of ADM into the subpanniculus pocket and the incision site (2-3 cm long) was sutured (Nylon 4/0, Ethicon, New Brunswick, NJ, USA). A light dressing was applied after disinfection of the surgical site with $70 \%$ alcohol and betadine. The implanted rats were observed for up to 6 months. We monitored inflammation and wound healing progress in detail. Moreover, randomly chosen five animals from each AlloDerm, SureDerm HD, and DermACELL groups were biopsied at 1,2,3, and 6 months. The biopsy area was tissue near the implanted dorsal region including epidermis, dermis, and posterior as well as anterior capsules.

Quantification of collagen deposition by histological assessment. For paraffin embedding, $10 \%$ paraformaldehyde was used for fixation of the biopsied tissue for $24 \mathrm{~h}$ before embedding in paraffin. The paraffin blocks were sliced into $4-\mu \mathrm{m}$ slices. According to type and period of implantation, 5 slices from each of the 5 randomly selected rats per each group were stained by H\&E to determine infiltration of cells and perform statistical analysis. Collagen fiber production and collagen intensity were evaluated by Masson's trichrome stain and quantified by using ImageJ software. 


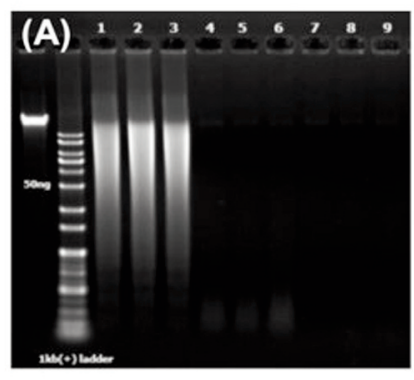

(B)

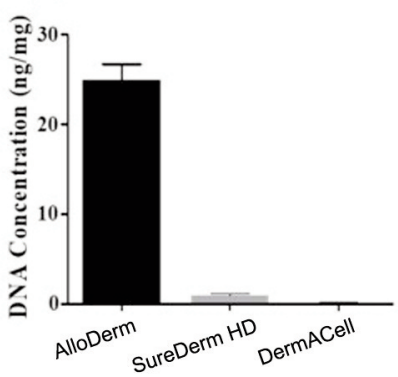

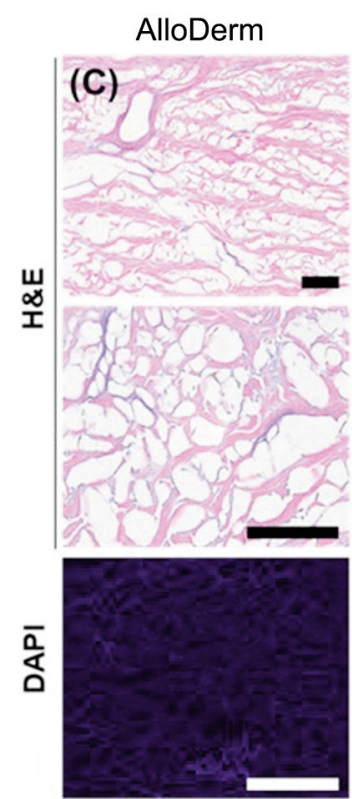
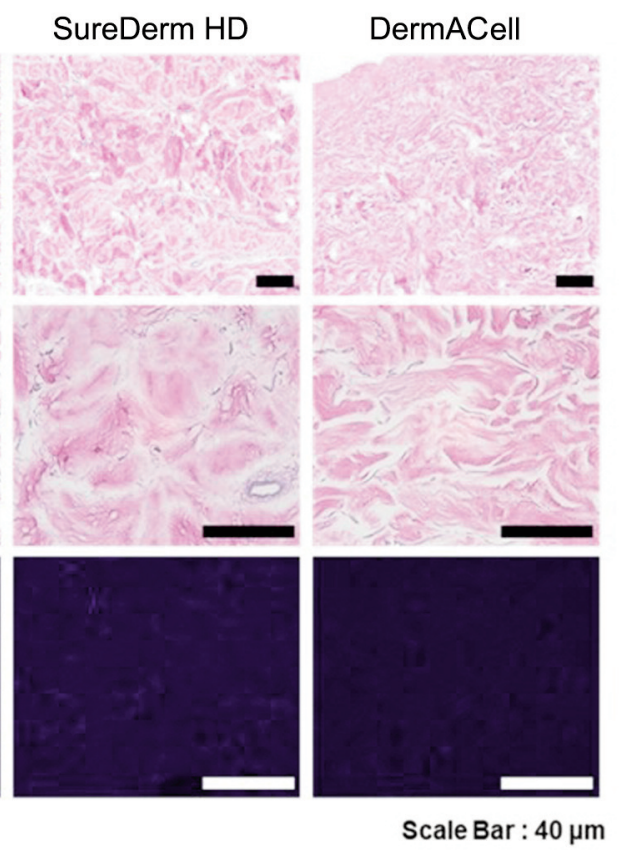

Figure 1. Decellularization assessment of acellular dermal matrices (ADMs). (A) The remaining double stranded DNA (dsDNA) in ADMs was analyzed by $1 \%$ agarose gel electrophoresis and staining with ethidium bromide; Lines 1-3 present three independent samples of AlloDerm; Lines 4 -6 present three independent samples of SureDerm HD; Lines 7-9 present three independent samples of DermACELL. (B) PicoGreen assay to measure dsDNA concentration. (C) $H \& E$ and DAPI staining to probe for intact cells and remnant nucleic acid, respectively.

Western blot analysis. The western blot analysis was conducted for MMP-2, MMP-3 and MMP-9 expression. The implants collected at 1-, 2-, 3-, and 6-months following implantation were lysed in PROPREPTM Protein Extraction Solution (C/T) (iNtRON, Seongnam, Republic of Korea) according to the manufacturer's protocol. A standard BCA method was used to determine protein concentration and subsequently, the protein samples were prepared for western blot. The same amount of protein was loaded on $12 \%$ SDS-PAGE gel, and then the proteins were transferred onto the blotting membrane. Membrane blocking was performed using 5\% of skim milk in the TBST buffer. Mouse anti-MMP2 antibody $(1: 1000$; Abcam; Cat\# ab86607), mouse anti-MMP9 antibody (1:1000; Abcam; Cat\#. ab38898), mouse anti-MMP3 antibody (1:1000; Abcam; Cat\# ab52915), mouse anti-TIMP1 antibody (1:1000; Abcam; Cat\#. ab61224), rabbit anti-TGF beta 1 antibody (1:1000; Abcam; Cat\# ab92486), mouse anti $\alpha$-SMA antibody (1:1000; Abcam; Cat\# ab7817) were used as primary antibodies and incubated with the membranes overnight at $40 \mathrm{C}$. Subsequently, goat anti mouse IgG H\&L (HPP) (1:5000; Abcam; Cat\# ab6789) secondary antibody was used. The level of protein expression was measured by an image analyzer (Las-3000, Photo Fuji, Tokyo, Japan). The images were acquired using Image Lab Touch (BioRad, Berkeley, CA, USA).

Statistical analysis. The mean values and \pm SEM obtained by Image J are shown in all figures. Prism statistical software (GraphPad Software, San Diego, CA, USA) was used for the statistical analysis. The statistical significance was determined using an unpaired $t$-test. Statistical significance was set at $p<0.05$.

\section{Results}

Decellularization evaluation of ADMs. The efficacy of the process of decellularization for AlloDerm, SureDerm HD, and DermACELL was evaluated by DNA fragment analysis using agarose gel electrophoresis (Figure 1A). AlloDerm contained large DNA fragments (>1,000 bp), however SureDerm HD and DermACELL were not found to contain large DNA fragments ( $>1,000 \mathrm{bp}$ ) (Figure 1A). The DNA content in AlloDerm was $24.94 \pm 2.17 \mathrm{ng} / \mathrm{mg}$, whereas SureDerm HD and DermACELL contained $0.93 \pm 0.25 \mathrm{ng} / \mathrm{mg}$ and $0.03 \pm 0.01$ $\mathrm{ng} / \mathrm{mg}$, respectively (Figure 1B). Furthermore, H\&E staining showed a lack of hematoxylin-stained nuclei in SureDerm HD and DermACELL indicating efficient cell removal, while AlloDerm showed dense darkly stained cell nuclei (Figure 1C). DAPI staining revealed complete removal of intact nuclei and remaining nuclear DNA in decellularized SureDerm HD and DermACELL as compared to AlloDerm, which showed heavy nuclear staining throughout the tissue (Figure 1C). These results suggest that SureDerm HD and DermACELL are appropriate for use in breast reconstruction.

Biochemical properties. A consideration when utilizing this biomaterial is whether it retains growth factors after the decellularization process, which support the activity of recipient 


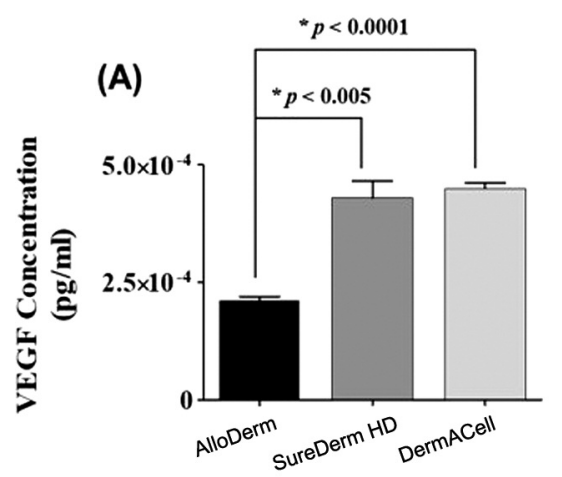

(B)

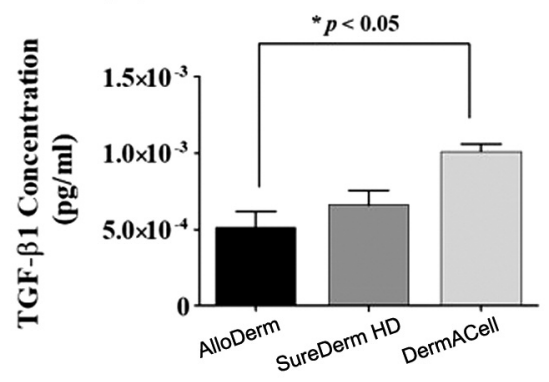

(C)

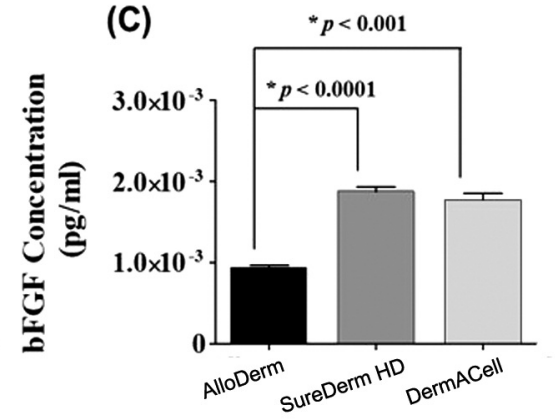

Figure 2. Biochemical properties. ELISA was used to quantify the growth factors retained in the acellular dermal matrices. (A) VEGF, (B) hTGF$\beta 1$, and $(C)$ bFGF. The data are shown as the mean value \pm SEM of three independent experiments.

cells, leading to better incorporation and recovery. The biochemical properties of AlloDerm, SureDerm HD, and DermACELL were analyzed by examining the levels of VEGF, TGF- $\beta 1$, and bFGF using established ELISA methods. SureDerm HD and DermACELL contained more VEGF, TGF$\beta 1$ and bFGF than AlloDerm (Figure 2A-C). VEGF content in SureDerm HD and DermACELL was found to be about 2.0-fold and 2.2-fold higher compared with AlloDerm, respectively (Figure 2A). The protein levels of TGF- $\beta 1$ in SureDerm HD and DermACELL inceased about 1.3-fold and 2.0-fold compared to AlloDerm, respectively (Figure 2B). In addition, SureDerm HD and DermACELL contained significantly increased levels of bFGF, approximately 2.0-fold and 1.9-fold, compared to AlloDerm, respectively (Figure 2C). Overall, the levels of the remaining growth factors after decellularization were higher in SureDerm HD and DermACELL and, therefore, may support recovery after implantation more efficiently than AlloDerm.

Cell adhesion and infiltration on ADMs. To confirm the capability of AMDs to support cell growth after implantation into body, we quantified cell confluence and cell infiltration using NIH 3T3 mouse fibroblasts (Figure 3A). ADMs were seeded with fibroblasts for up to 7 days. Fibroblasts cultured on AlloDerm were unevenly distributed on the surface of the dermal matrix showing no increase in cell number (Figure $3 \mathrm{~B})$. Fibroblasts were adherent and spread on the surface of SureDerm HD and DermACELL at day 7 (Figure 3B). A confluent fibroblastic monolayer lining SureDerm HD surface was noticed (Figure 3B), however few cells were observed inside the SureDerm HD at this time (Figure 3B). A higher percentage of cells was observed not only on the surface of DermACELL but also inside the ADM compared to AlloDerm and SureDerm HD (Figure 3B). The surface of DermACELL was more readily infiltrated by fibroblasts than that of the other two ADMs. These results indicated that SureDerm HD and DermACELL were more cytocompatible than AlloDerm for cell adhesion and infiltration (Figure 3B).
Histologic analysis of implanted ADMs. In vivo evaluation of each ADM material for bilateral breast reconstruction was performed in a rat model (Figure 4). And the result of host remodeling response was confirmed through histomorphologic observation as a function of time and location of the explants. After implantation of AlloDerm, SureDerm HD, or DermACELL, all AMDs were well accepted and were not harmful to living tissue. Moreover, no infection and no graft rejection were observed at different time points after implantation of these materials (Figure 5A). After implantation of the porous architecture of AlloDerm, SureDerm HD, and DermACELL, vascular and connective tissue was formed and developed within ADMs over time (Figure 5A). SureDerm HD and DermACELL exhibited mild inflammation with immune cell infiltration and extracellular matrix (ECM) deposition compared to the AlloDerm (Figure 5B). The infiltration level of immune cells in DermACELL (26.20\% \pm 9.94$)$ was lower than that in SureDerm HD $(54.80 \% \pm 10.65)$ and AlloDerm $(81.40 \% \pm 7.67)$ at 1 month after implantation (Figure 5B). After 2 months of implantation, the infiltration level of immune cells significantly increased in AlloDerm $(140.8 \% \pm 4)$ compared to the first month. In contrast, it was decreased in both of SureDerm HD $(38.00 \% \pm 6.55)$ and DermACELL $(24.20 \% \pm 5.67)$ compared to the first month. Moreover, fibroblasts were observed in all ADMs at 1 month after implantation and the infiltration levels of fibroblasts in all ADMs were gradually increased over time. Specially, the infiltration level of fibroblasts in SureDerm HD and DermACELL revealed a 2 fold and 3-fold increase at 1 month compared to the AlloDerm (Figure 5C). However, the infiltration of fibroblasts in all AMDs was decreased after 6 months of implantation (Figure 5C).

Evaluation of collagen content of implanted ADMs. The content and distribution pattern of collagen, an important structural component of ADMs, were evaluated by MT staining. Quantification of collagen density showed that the amounts of collagen in AlloDerm, SureDerm HD, and DermACELL were 

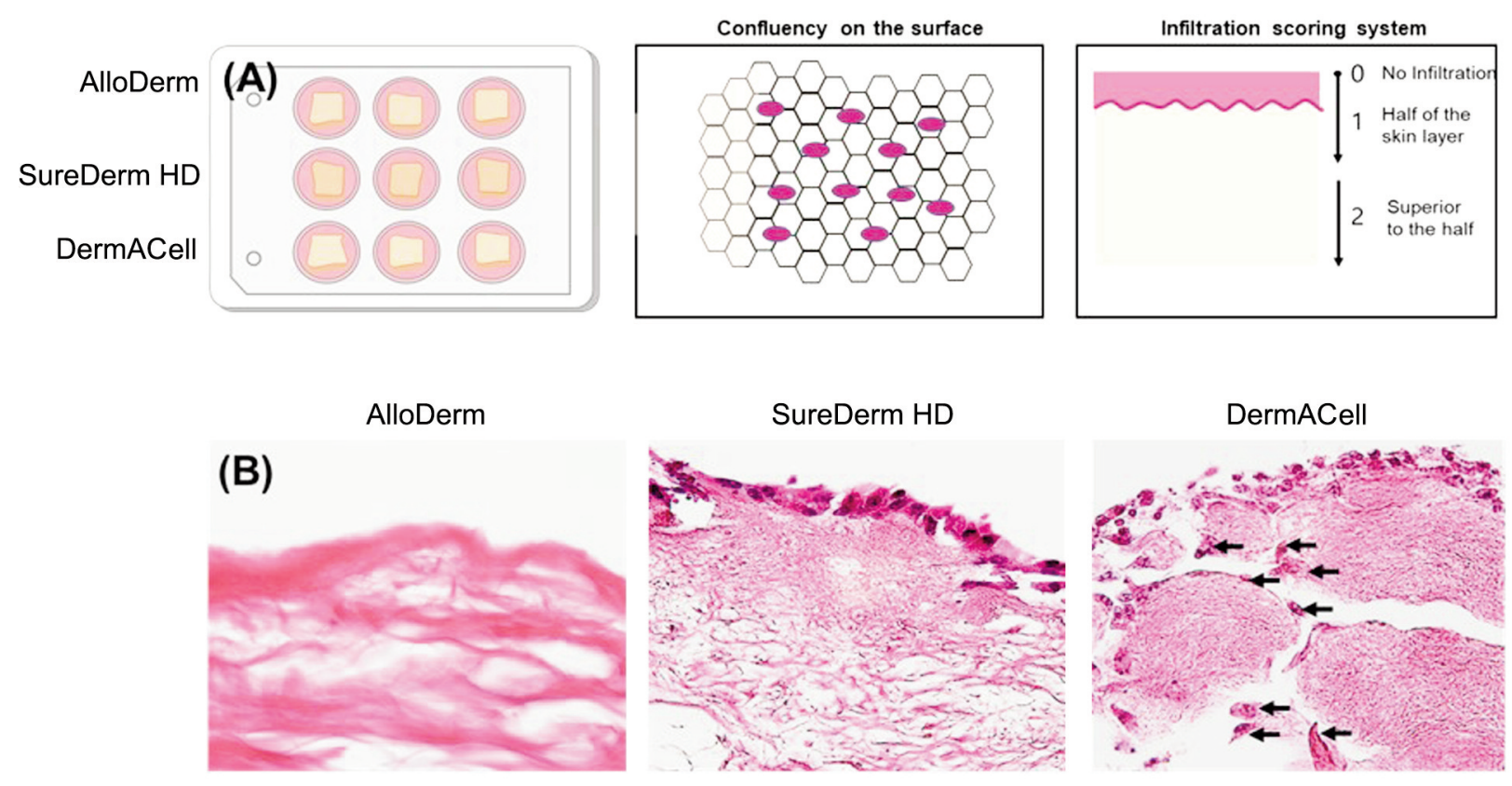

Figure 3. Cytocompatibility with fibroblasts. (A) Schematic representation of cell adhesion and infiltration into the acellular dermal matrices (ADMs) in vitro. (B) H\&E staining of ADMs cultured with cells. NIH 3 T3 fibroblasts were seeded on the surface of ADMs and cultured for 7 days. Invasion of fibroblasts on the dermal surface is shown by arrows. The experiment was performed in triplicate.

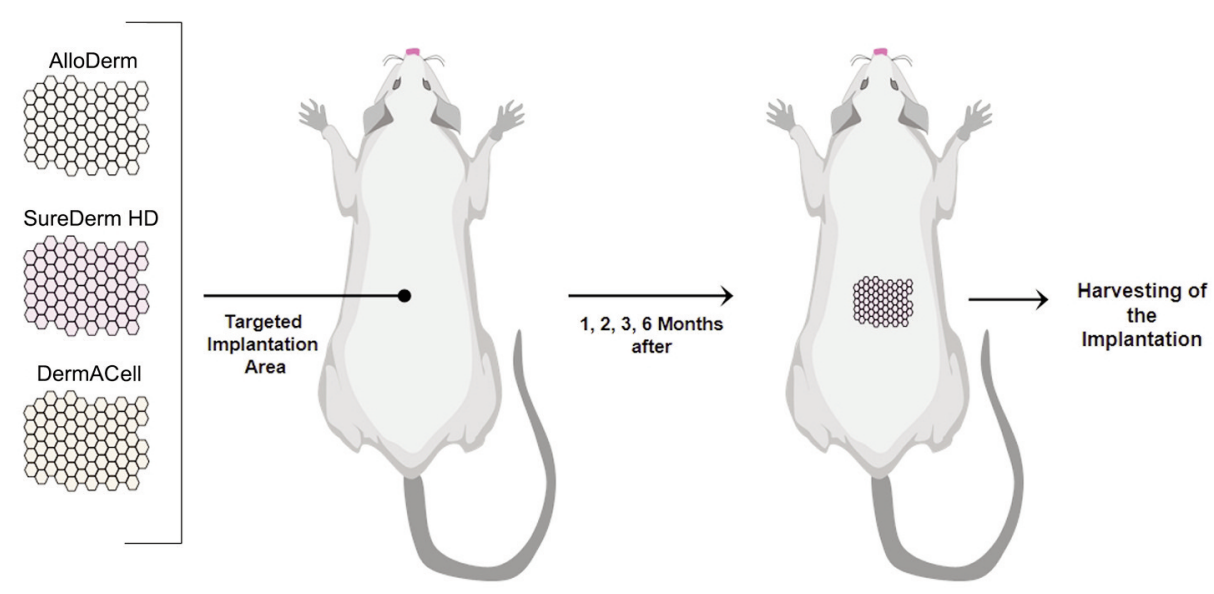

Figure 4. Schematic diagram of the in vivo experiment.

significantly increased and their porous architecture was replaced by new collagen over time (Figure 6A). After decellularization, small amounts of collagen remain on ADMs. However, noumerous collagen fibers appeared over time and the porous architecture of the ADM was fully restored by new collagen fibers after 6 months of implantation (Figure 6A).
Collagen deposition within the implanted SureDerm HD and DermACELL was significantly increased approximately $64.8 \%$ and $55.2 \%$ at 1 month, $40.9 \%$ and $54.5 \%$ at 2 months, $7.6 \%$ and $47.1 \%$ at 3 months, and $38 \%$ and $35.8 \%$ at 6 months compared to AlloDerm after implantation, respectively $(p<0.05$, Figure 6B). 


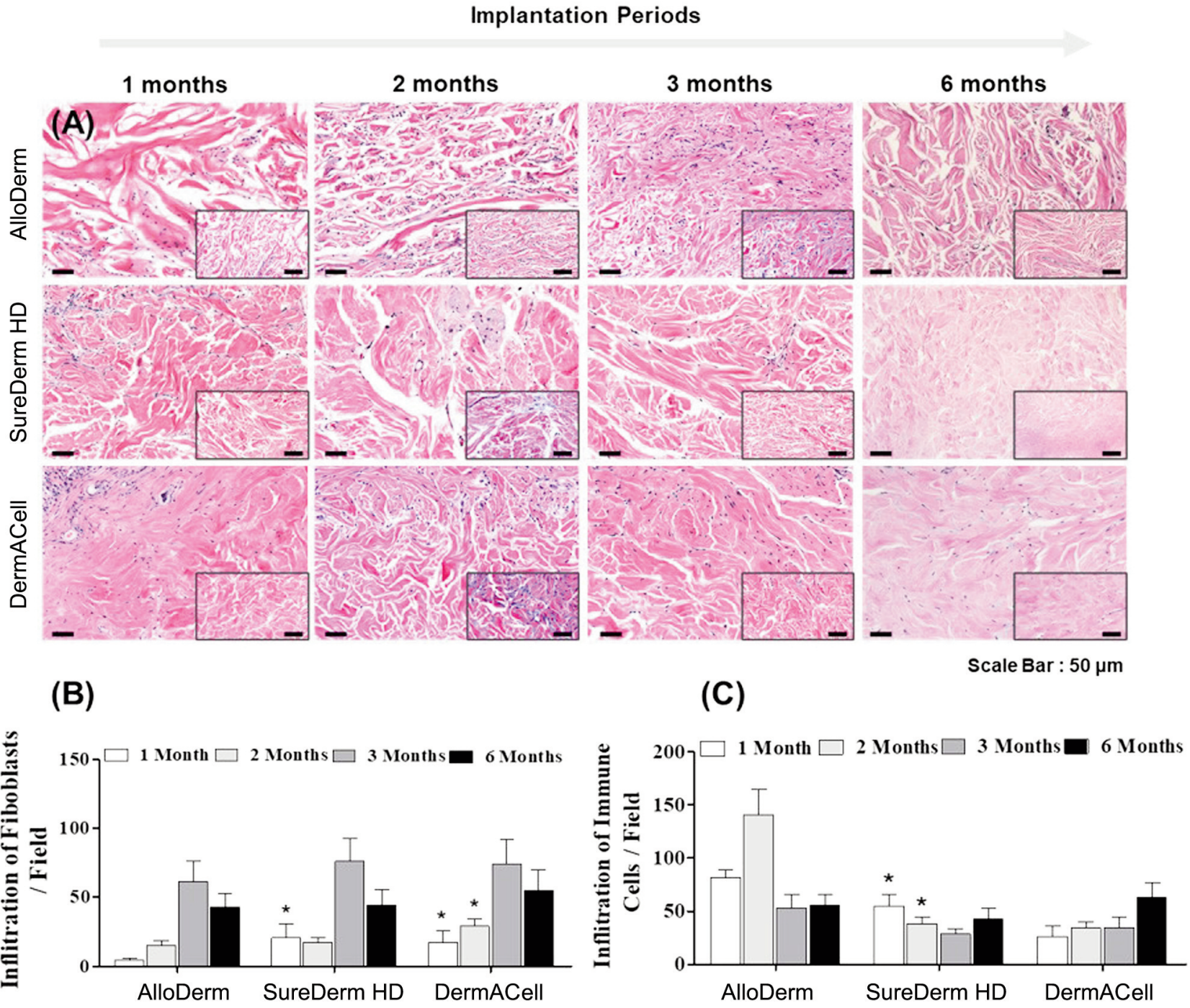

Figure 5. Tissue suitability assessment. (A) Host tissue ingrowth and cellular infiltration of implanted acellular dermal matrices (ADMs) over time were analyzed by $H \& E$ staining $(\times 200$, scale bar $=50 \mu \mathrm{m})$. (B) Infiltration of immune cells $(C)$ Infiltration of fibroblasts. The data is shown as the mean value \pm SEM of five independent experiments. $p<0.05$; significantly different from the AlloDerm.

Effect of ADMs on the expression of MMPs. MMPs are a family of enzymes that include at least 28 members, most of which degrade ECM. Analysis of MMPs by western blotting showed differential expression of these proteins at different time points. The expression and activities of MMP-2 were increased during the acute phase of wound and decreased during the recovery stage. As depicted in Figure 7A, clear bands were detected for MMP-2, thereby indicating that this MMP is expressed in ADMs. The differences in MMP-2 among all groups were not statistically significant at 3 months after implantation ( $\mathrm{p}>0.05$ ). The MMP-2 levels in AlloDerm were the highest and that in SureDerm HD were the lowest among the three groups at 6 months after implantation (Figure
7B). The differences in MMP-3 among all groups were not statistically significant at 6 months after implantation $(\mathrm{p}>0.05)$ (Figure 7C). The MMP-9 levels in AlloDerm were the highest and those of SureDerm HD and DermACELL were lower at 6 months as shown in Figure 7A and D. These results suggest that expression of proteins involved in ECM remodelling in SureDerm HD is delayed, compared AlloDerm and DermACELL during wound healing process.

\section{Discussion}

In this study, we investigated the histological and biochemical characteristics of the novel human derived SureDerm HD, 


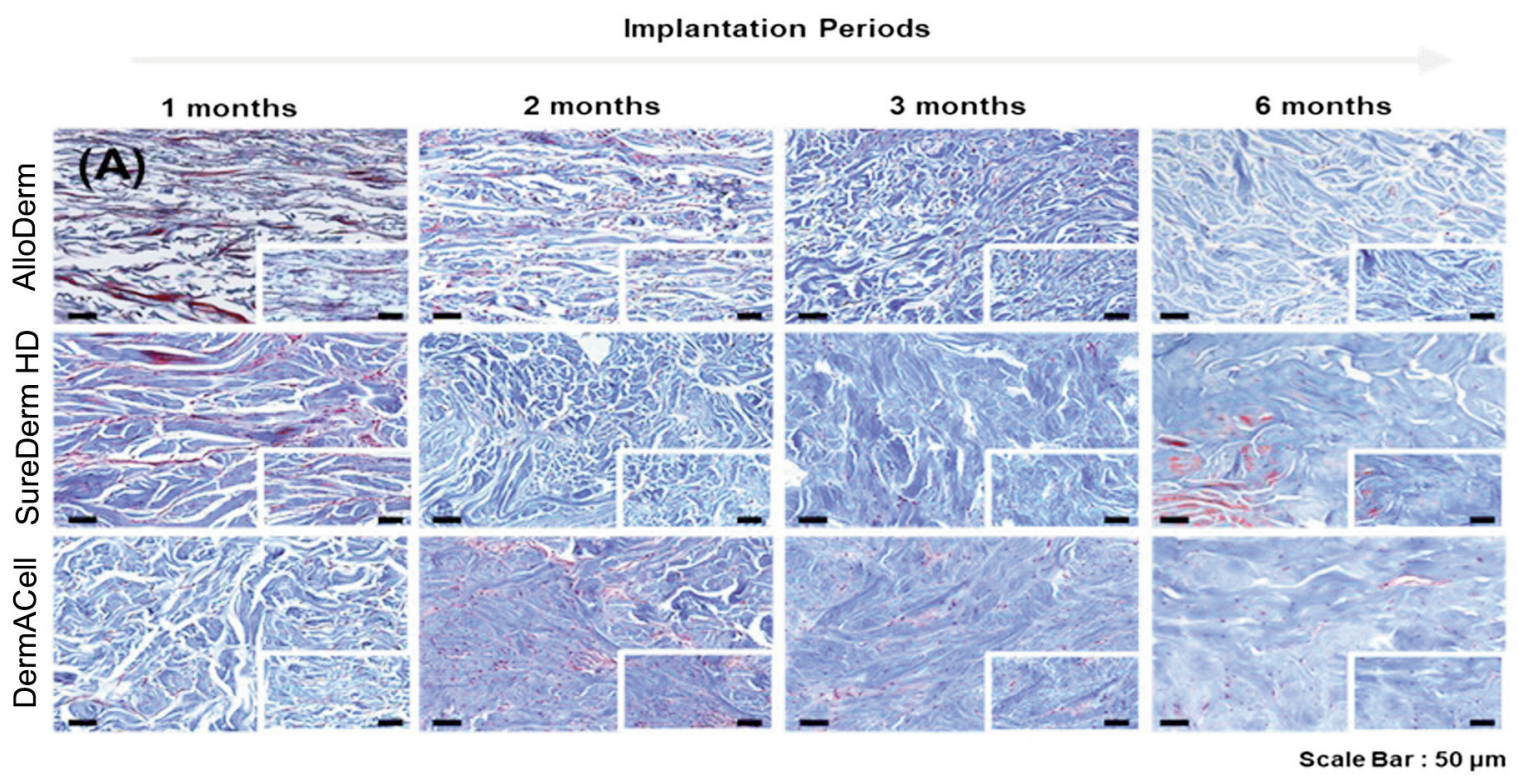

(B)

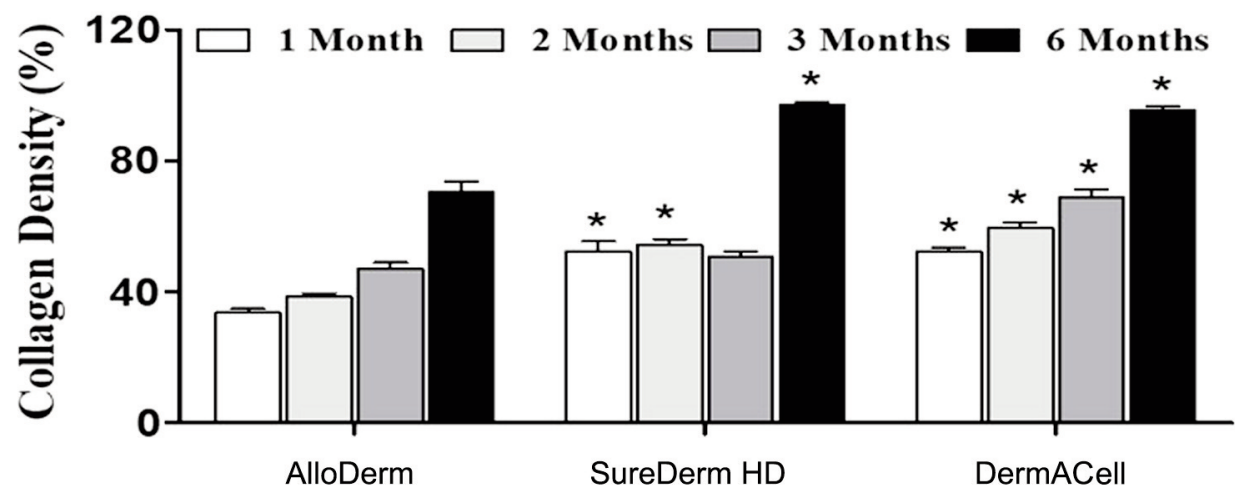

Figure 6. Histological analysis of collagen deposition after acellular dermal matrix implantation was analyzed by Masson's trichrome stain. The data is shown as the mean value \pm SEM of five independent experiments. $p<0.05$; significantly different from the AlloDerm.

which is produced through a unique process, and compared them with those of two commercial ADM products.

The process of development and manufacturing of human ADMs consists of decellularization, cross-linking, preservation, and sterility assurance $(24,25)$. Decellularization performed through chemical, physical, biological, or combinatorial methods plays the most important role in all phases. The products of each method are distinctive. The elimination of all immunogenic factors from donor tissue and antigenic materials such as cells, cell debris and chromosome fragments while retaining the ECM biochemistry and structure are the goals of decellularization process. The ECM, which retains dermal collagen architecture, has an important role (26). Moreover, the inflammatory response is caused by the existence of residual DNA in biological scaffold materials (27). H\&E staining, DAPI staining and evaluation of DNA samples on agarose gel electrophoresis indicated that the human derived SureDerm HD was completely decellularized compared with AlloDerm and DermACELL. In addition, essential growth factors promoting hyaluronan production, through an increase in the mRNA levels of hyaluronic acid synthase 2 , depend on bFGF (28). In the present study, we found that SureDerm HD contains significant levels of TGF- $\beta 1$, VEGF, and bFGF. TGF- $\beta 1$ and VEGF stimulate cell proliferation whereas bFGF is an effective inducer of hyaluronic acid production. Furthermore, the tensile and elasticity of AMD are contributed by collagen and elastin fibers, which are the main components of this material. Therefore, it can be considered that SureDerm HD has a good biological behavior and immunological safety during the implantation process.

When breast is reconstructed by implanting ADMs, it is recolonized by fibroblasts, myofibroblasts and cells of the 


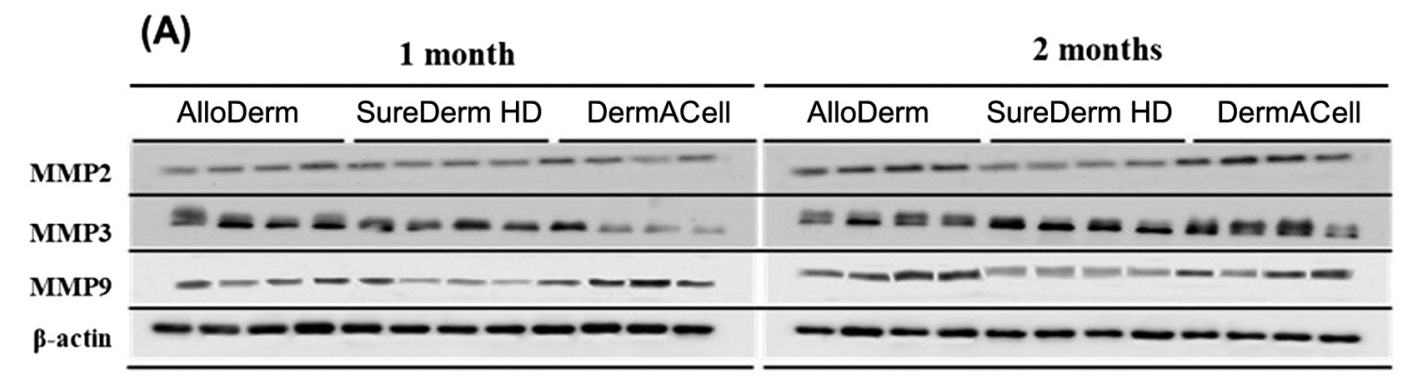

3 months

6 months

\begin{tabular}{|c|c|c|c|c|c|}
\hline \multirow[b]{2}{*}{ MMP2 } & SureDerm HD & DermACell & AlloDerm & SureDerm HD & DermACell \\
\hline & \multicolumn{2}{|c|}{$--\cdots-1-\cdots=$} & \multicolumn{3}{|c|}{$----\div-1-\cdots$} \\
\hline MMP3 & 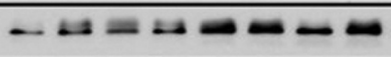 & $\Delta \infty a \mathrm{ab}$ & \multicolumn{3}{|c|}{$--\cdots--\infty \cdots$} \\
\hline MMP9 & $---\cdots--\div$ & --- & \multicolumn{3}{|c|}{$---1-1-1-1-2$} \\
\hline$\beta$-actin & $-\cdots-1$ & $-\infty-\infty$ & \multicolumn{3}{|c|}{$-\cdots---\cdots-\cdots$} \\
\hline
\end{tabular}

(B)

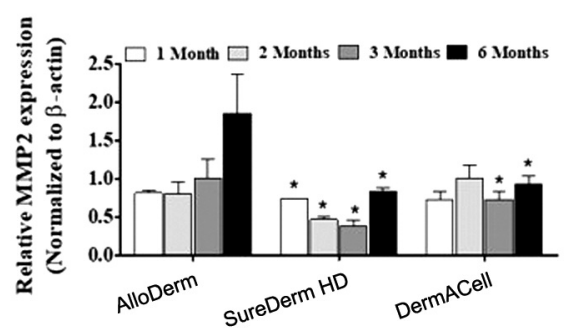

(C)

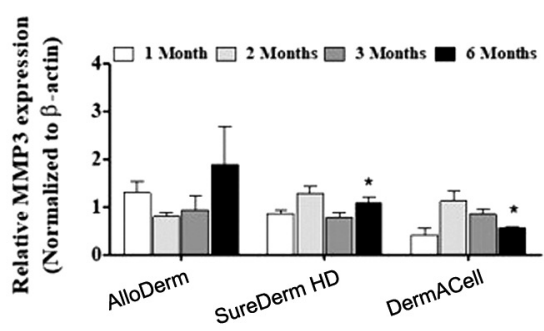

(D)

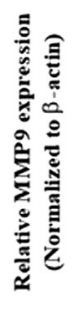

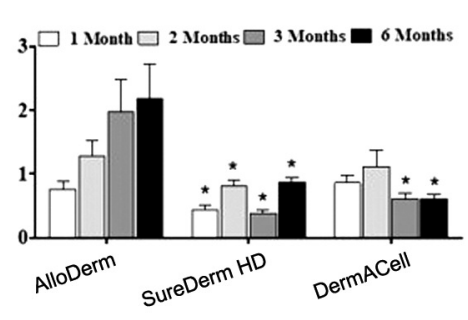

Figure 7. Remodeling related protein expression in implanted acellular dermal matrices was evaluated by western blot. The data represent the mean value \pm SEM of four independent experiments. $p<0.05$; significantly different from the AlloDerm.

immune system namely lymphocytes, macrophages, granulocytes, and mast cells (12-29). The fibrosis and neovascularization processes establish capsule and capillaries as well as lymphomagenesis. Therefore, AMDs biocompatible with host tissue, activate biologic responses as adhesion and proliferation of host cells without cytotoxicity (30). Based on our results of increased infiltration of fibroblasts, cell adhesion, and morphological stability, we highly recommend SureDerm HD as a good product for employing in breast reconstruction.

The phases of wound healing consist of haemostasis, inflammation, proliferation, deposition and remodeling of ECM that affect tissue integration after AMD implantation (11, 31-33). In our study, histological analysis showed increased deposition of collagen fibers over time. Especially, the intensity of collagen at sixth months was approximately double compared with that at 1 month after implantation. This result suggests rapid cellular infiltration of the implant. The loosely organized collagen of ADMs was replaced by newly synthesized collagen at 6 months of implantation.
Additionally, elastin fiber deposition was gradually increased only in DermACELL during the observed time, whereas it was decreased over time in both SureDerm HD and AlloDerm. These results may be due to the method of decellularization.

MMPs are enzymes belonging to the family of metalloendopeptidases that contribute to all phases of the wound healing process by regulating immune cell influx, facilitating migration of fibroblasts, ketatinocytes and scar tissue reconstruction $(34,35)$. In addition, they play a critical role in the remodeling of ECM components. Normally, throughout the process of wound healing, the levels of MMPs increase in an acute wound and decrease as wound healing occurs. MMP-2 (gelatinase A, 72-kDa gelatinase) can degrade several matrix components including collagen types I, IV, V, VII, XI, and laminin (36). MMP-3 has the ability to hydrolyse collagen types II, III, IV, IX and laminin, while MMP9 (gelatinase B, $72 \mathrm{kDa}$ ) is able to degrade collagen types III, IV, V, XIV and elastin $(37,38)$. In our study, AlloDerm showed dramatically increased levels of 
MMP-2, MMP-3, and MMP-9 at 6 months after implantation compared with those of SureDerm HD and DermACELL $(p<0.05)$. These results suggest that SureDerm HD could provide long-term structural integrity, and result in prolonged remodeling.

In summary, our study focused on the three ADMs for breast reconstructive application and evaluated their performance through in vitro and in vivo studies. Among them, SureDerm HD showed long term structural integrity and provided a more favorable environment for cell infiltration and ECM deposition. Besides, protein expression results demonstrated SureDerm HD had a significant remodeling effect on the wound site during the healing stage. based on our data, SureDerm HD could be a potential implant in clinical applications for breast reconstruction.

\section{Conflicts of Interest}

The Authors declare no conflicts of interest in relation to this study.

\section{Authors' Contributions}

Conceptualization, S.Y.N. and C.Y.H.; methodology, P.N.C.; validation, P.N.C. and X.R.Z.; investigation, P.N.C., D.N., O.F., and X.R.Z.; data curation, P.N.C. and S.Y.N; writing - original draft preparation, P.N.C. and S.Y.N.; writing-review and editing, P.N.C., O.F., and S.Y.N.; supervision, S.Y.N. and C.Y.H.; project administration, C.Y.H. All Authors have read and agreed to the published version of the manuscript.

\section{Acknowledgements}

This work was supported by grant No. 14-2017-019 from SNUBH research Fund and HansBiomed.

\section{References}

1 Wainwright DJ: Use of an acellular allograft dermal matrix (AlloDerm) in the management of full-thickness burns. Burns 21(4): 243-248, 1995. PMID: 7662122. DOI: 10.1016/03054179(95)93866-i

2 Butler CE, Langstein HN and Kronowitz SJ: Pelvic, abdominal, and chest wall reconstruction with AlloDerm in patients at increased risk for mesh-related complications. Plast Reconstr Surg 116(5): 1263-75; discussion 1276-7, 2005. PMID: 16217466. DOI: 10.1097/01.prs.0000181692.71901.bd

3 Chaplin JM, Costantino PD, Wolpoe ME, Bederson JB, Griffey ES and Zhang WX: Use of an acellular dermal allograft for dural replacement: an experimental study. Neurosurgery 45(2): 320-327, 1999. PMID: 10449077. DOI: 10.1097/00006123-19990800000025

4 Tal H: Subgingival acellular dermal matrix allograft for the treatment of gingival recession: a case report. J Periodontol 70(9): 1118-1124, 1999. PMID: 10505815. DOI: 10.1902/jop.1999.70. 9.1118

5 Sclafani AP, Romo T 3rd, Jacono AA, McCormick S, Cocker R and Parker A: Evaluation of acellular dermal graft in sheet
(AlloDerm) and injectable (micronized AlloDerm) forms for soft tissue augmentation. Clinical observations and histological analysis. Arch Facial Plast Surg 2(2): 130-136, 2000. PMID: 10925439. DOI: 10.1001/archfaci.2.2.130

6 An G, Walter RJ and Nagy K: Closure of abdominal wall defects using acellular dermal matrix. J Trauma 56(6): 1266-1275, 2004. PMID: 15211136. DOI: 10.1097/01.ta.0000068241.66186.00

7 Baxter RA: Intracapsular allogenic dermal grafts for breast implant-related problems. Plast Reconstr Surg 112(6): 1692-6; discussion 1697-8, 2003. PMID: 14578804. DOI: 10.1097/ 01.PRS.0000086365.25453.C3

8 Buinewicz B and Rosen B: Acellular cadaveric dermis (AlloDerm): a new alternative for abdominal hernia repair. Ann Plast Surg 52(2): 188-194, 2004. PMID: 14745271. DOI: 10.1097/01.sap.0000100895.41198.27

9 Bonnans C, Chou J and Werb Z: Remodelling the extracellular matrix in development and disease. Nat Rev Mol Cell Biol 15(12): 786-801, 2014. PMID: 25415508. DOI: 10.1038/nrm3904

10 Cummings LC, Kaldahl WB and Allen EP: Histologic evaluation of autogenous connective tissue and acellular dermal matrix grafts in humans. J Periodontol 76(2): 178-186, 2005. PMID: 15974840. DOI: 10.1902/jop.2005.76.2.178

11 Carlson TL, Lee KW and Pierce LM: Effect of cross-linked and non-cross-linked acellular dermal matrices on the expression of mediators involved in wound healing and matrix remodeling. Plast Reconstr Surg 131(4): 697-705, 2013. PMID: 23249980. DOI: 10.1097/PRS.0b013e3182818a3d

12 Boháč M, Danišovič L, Koller J, Dragúňová J and Varga I: What happens to an acellular dermal matrix after implantation in the human body? A histological and electron microscopic study. Eur J Histochem 62(1): 2873, 2018. PMID: 29569868. DOI: 10.4081/ejh.2018.2873

13 Carlsson A, Gronet E, Rose L and Chan R: Clinical applications of acellular dermal matrices in reconstructive surgery. Skin Tissue Engineering and Regenerative Medicine: 109-124, 2020. DOI: 10.1016/B978-0-12-801654-1.00006-1

14 Vorotnikova E, McIntosh D, Dewilde A, Zhang J, Reing JE, Zhang L, Cordero K, Bedelbaeva K, Gourevitch D, Heber-Katz E, Badylak SF and Braunhut SJ: Extracellular matrix-derived products modulate endothelial and progenitor cell migration and proliferation in vitro and stimulate regenerative healing in vivo. Matrix Biol 29(8): 690-700, 2010. PMID: 20797438. DOI: 10.1016/j.matbio.2010.08.007

15 Carruthers CA, Dearth CL, Reing JE, Kramer CR, Gagne DH, Crapo PM, Garcia O Jr, Badhwar A, Scott JR and Badylak SF: Histologic characterization of acellular dermal matrices in a porcine model of tissue expander breast reconstruction. Tissue Eng Part A 21(1-2): 35-44, 2015. PMID: 24941900. DOI: 10.1089/ten.TEA.2014.0095

16 Breuing $\mathrm{KH}$ and Warren SM: Immediate bilateral breast reconstruction with implants and inferolateral AlloDerm slings. Ann Plast Surg 55(3): 232-239, 2005. PMID: 16106158. DOI: 10.1097/01.sap.0000168527.52472.3c

17 Spear SL, Parikh PM, Reisin E and Menon NG: Acellular dermisassisted breast reconstruction. Aesthetic Plast Surg 32(3): 418-425, 2008. PMID: 18338102. DOI: 10.1007/s00266-008-9128-8

18 Salzberg CA: Nonexpansive immediate breast reconstruction using human acellular tissue matrix graft (AlloDerm). Ann Plast Surg 57(1): 1-5, 2006. PMID: 16799299. DOI: 10.1097/01.sap. $0000214873.13102 .9 \mathrm{f}$ 
19 Bindingnavele V, Gaon M, Ota KS, Kulber DA and Lee DJ: Use of acellular cadaveric dermis and tissue expansion in postmastectomy breast reconstruction. J Plast Reconstr Aesthet Surg 60(11): 1214-1218, 2007. PMID: 17459797. DOI: 10.1016/ j.bjps.2007.03.015

20 Crapo PM, Gilbert TW and Badylak SF: An overview of tissue and whole organ decellularization processes. Biomaterials 32(12): 3233-3243, 2011. PMID: 21296410. DOI: 10.1016/ j.biomaterials.2011.01.057

21 Brown BN, Valentin JE, Stewart-Akers AM, McCabe GP and Badylak SF: Macrophage phenotype and remodeling outcomes in response to biologic scaffolds with and without a cellular component. Biomaterials 30(8): 1482-1491, 2009. PMID: 19121538. DOI: 10.1016/j.biomaterials.2008.11.040

22 Badylak SF, Valentin JE, Ravindra AK, McCabe GP and Stewart-Akers AM: Macrophage phenotype as a determinant of biologic scaffold remodeling. Tissue Eng Part A 14(11): 18351842, 2008. PMID: 18950271. DOI: 10.1089/ten.tea.2007.0264

23 Keane TJ, Londono R, Turner NJ and Badylak SF: Consequences of ineffective decellularization of biologic scaffolds on the host response. Biomaterials 33(6): 1771-1781, 2012. PMID: 22137126. DOI: $10.1016 /$ j.biomaterials.2011.10.054

24 Badylak SF, Freytes DO and Gilbert TW: Extracellular matrix as a biological scaffold material: Structure and function. Acta Biomater 5(1): 1-13, 2009. PMID: 18938117. DOI: 10.1016/ j.actbio.2008.09.013

25 Gilbert TW: Strategies for tissue and organ decellularization. J Cell Biochem 113(7): 2217-2222, 2012. PMID: 22415903. DOI: $10.1002 /$ jcb. 24130

26 Bush $\mathrm{K}$ and Gertzman A: Process development and manufacturing of human and animal acellular dermal matrices. Skin Tissue Engineering and Regenerative Medicine: 83-108, 2018. DOI: $10.1016 / \mathrm{B} 978-0-12-801654-1.00005-\mathrm{X}$

27 Moyer HR, Hart AM, Yeager J and Losken A: A histological comparison of two human acellular dermal matrix products in prosthetic-based breast reconstruction. Plast Reconstr Surg Glob Open 5(12): e1576, 2017. PMID: 29632762. DOI: 10.1097/ GOX.0000000000001576

28 Ban MJ, Park JH, Kim JW, Park KN, Lee JY, Kim HK and Lee SW: The efficacy of Fibroblast growth factor for the treatment of chronic vocal fold scarring: From animal model to clinical application. Clin Exp Otorhinolaryngol 10(4): 349-356, 2017. PMID: 27671715. DOI: $10.21053 /$ ceo.2016.00941

29 Garcia O Jr and Scott JR: Analysis of acellular dermal matrix integration and revascularization following tissue expander breast reconstruction in a clinically relevant large-animal model. Plast Reconstr Surg 131(5): 741e-751e, 2013. PMID: 23629113. DOI: $10.1097 /$ PRS.0b013e3182865c6d
$30 \mathrm{Ng} \mathrm{KW}$, Khor HL and Hutmacher DW: In vitro characterization of natural and synthetic dermal matrices cultured with human dermal fibroblasts. Biomaterials 25(14): 2807-2818, 2004. PMID: 14962559. DOI: 10.1016/j.biomaterials.2003.09.058

31 Kirk JF, Ritter G, Finger I, Sankar D, Reddy JD, Talton JD, Nataraj C, Narisawa S, Millán JL and Cobb RR: Mechanical and biocompatible characterization of a cross-linked collagenhyaluronic acid wound dressing. Biomatter 3(4): e25633, 2013. PMID: 23896569. DOI: 10.4161/biom.25633

32 Anderson JM, Rodriguez A and Chang DT: Foreign body reaction to biomaterials. Semin Immunol 20(2): 86-100, 2008. PMID: 18162407. DOI: 10.1016/j.smim.2007.11.004

33 Philandrianos C, Andrac-Meyer L, Mordon S, Feuerstein JM, Sabatier F, Veran J, Magalon G and Casanova D: Comparison of five dermal substitutes in full-thickness skin wound healing in a porcine model. Burns 38(6): 820-829, 2012. PMID: 22652473. DOI: $10.1016 /$ j.burns.2012.02.008

34 Armstrong DG and Jude EB: The role of matrix metalloproteinases in wound healing. J Am Podiatr Med Assoc 92(1): 12-18, 2002. PMID: 11796794. DOI: 10.7547/87507315-92-1-12

35 Auf dem keller U and Sabino F: Matrix metalloproteinases in impaired wound healing. Metalloproteinases In Medicine: 1, 2016. DOI: $10.2147 /$ MNM.S68420

36 Lindman JP, Talbert M, Zhang W, Powell B, Accortt NA and Rosenthal EL: Promotion of acellular dermal matrix resolution in vitro by matrix metalloproteinase- 2 . Arch Facial Plast Surg 8(3): 208-212, 2006. PMID: 16702534. DOI: 10.1001/archfaci.8.3.208

37 Murphy G, Cockett MI, Ward RV and Docherty AJ: Matrix metalloproteinase degradation of elastin, type IV collagen and proteoglycan. A quantitative comparison of the activities of 95 $\mathrm{kDa}$ and $72 \mathrm{kDa}$ gelatinases, stromelysins-1 and -2 and punctuated metalloproteinase (PUMP). Biochem J 277 (Pt 1): 277-279, 1991. PMID: 1649600. DOI: 10.1042/bj2770277

38 Shimokawa Ki K, Katayama M, Matsuda Y, Takahashi H, Hara I, Sato $\mathrm{H}$ and Kaneko S: Matrix metalloproteinase (MMP)-2 and MMP-9 activities in human seminal plasma. Mol Hum Reprod 8(1): 32-36, 2002. PMID: 11756567. DOI: 10.1093/molehr/8.1.32
Received June 25, 2021

Revised July 12, 2021

Accepted July 13, 2021 